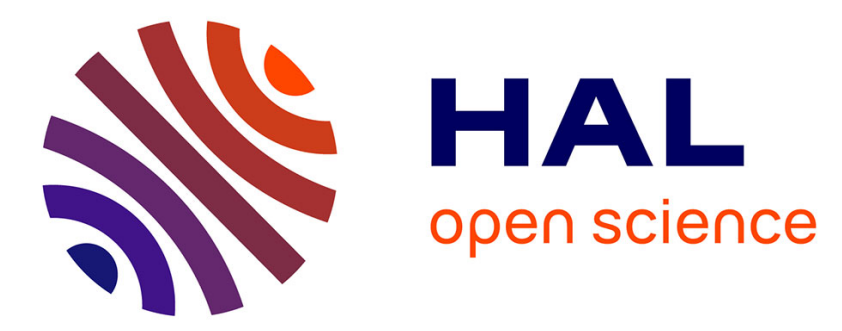

\title{
Effet de basses températures appliquées aux parties aérienne ou racinaire de la tomate sur l'absorption d'éléments minéraux et la fertilité pollinique
} Pierre Cornillon, Brigitte Maisonneuve, Magali Auge, Paul Florent

\section{- To cite this version:}

Pierre Cornillon, Brigitte Maisonneuve, Magali Auge, Paul Florent. Effet de basses températures appliquées aux parties aérienne ou racinaire de la tomate sur l'absorption d'éléments minéraux et la fertilité pollinique. Agronomie, 1985, 5 (1), pp.33-38. hal-00884730

\section{HAL Id: hal-00884730 \\ https://hal.science/hal-00884730}

Submitted on 1 Jan 1985

HAL is a multi-disciplinary open access archive for the deposit and dissemination of scientific research documents, whether they are published or not. The documents may come from teaching and research institutions in France or abroad, or from public or private research centers.
L'archive ouverte pluridisciplinaire HAL, est destinée au dépôt et à la diffusion de documents scientifiques de niveau recherche, publiés ou non, émanant des établissements d'enseignement et de recherche français ou étrangers, des laboratoires publics ou privés. 


\section{Effet de basses températures appliquées aux parties aérienne ou racinaire de la tomate sur l'absorption d'éléments minéraux et la fertilité pollinique}

Pierre CORNILLON \& Brigitte MAISONNEUVE $\left({ }^{*}\right)\left({ }^{1}\right)$

avec la collaboration de Magali AUGE \& Paul FLORENT

I.N.R.A., Station d'Agronomie

(*) Station d'Amélioration des Plantes maraîchères, Centre de Recherches d'Avignon, BP 91, F 84140 Montfavet

(1) Adresse actuelle : I.N.R.A., Station de Génétique et d'Amélioration des Plantes, Route de St-Cyr, F 78000 Versailles

Deux génotypes de tomate, "Montfavet $n^{\circ} 63-4$ » et «Coldset », cultivés en solution nutritive, ont été soumis à une combinaison de traitements thermiques : température de $7{ }^{\circ} \mathrm{C}-8{ }^{\circ} \mathrm{C}$ ou $13{ }^{\circ} \mathrm{C}-25^{\circ} \mathrm{C}$ (alternance nuitjour) pour la partie aérienne et de $8{ }^{\circ} \mathrm{C}$ ou $18{ }^{\circ} \mathrm{C}$ au niveau des racines.

Nous observons l'avortement du pollen lorsque la température de l'air ambiante est basse $\left(7^{\circ} \mathrm{C}-8{ }^{\circ} \mathrm{C}\right)$, quelle que soit la température des racines ; "Coldset » étant plus sensible au froid que "Montfavet $\mathrm{n}^{\circ} 63-4$ ". La consommation d'eau dépend essentiellement de la température de l'air avec une importante modulation liée à la température racinaire. Les deux génotypes ont un comportement très voisin.

Au contraire, l'absorption de l'ion $\mathrm{H}_{2} \mathrm{PO}_{4}^{-}$dépend uniquement de la température des racines, avec une absorption normale à $18{ }^{\circ} \mathrm{C}$ et une réduction d'environ $50 \mathrm{p}$. 100 des prélèvements à $8{ }^{\circ} \mathrm{C}$ pour les 2 génotypes. L'absorption de l'ion $\mathrm{NO}_{3}^{-}$et des cations est une fonction complexe de la température des racines et de celle de la partie aérienne. Pour les consommations des ions $\mathrm{NO}_{3}^{-}$et $\mathrm{K}^{+}$, nous notons une différence entre les 2 génotypes pour la température racinaire de $8{ }^{\circ} \mathrm{C}$. Il n'apparaît donc aucune relation entre l'avortement du pollen au froid, qui dépend seulement de la température de l'air ambiante, et l'absorption minérale, qui est tributaire également de la température du milieu racinaire.

Mots clés additionnels : Lycopersicon esculentum Mill., nutrition hydrique, nutrition minérale, qualité du pollen.

Two tomato genotypes, "Montfavet $\mathrm{n}^{\circ}$ 63-4" and "Coldset", were cultivated in nutrient solution with temperature treatments of $7^{\circ} \mathrm{C}-8{ }^{\circ} \mathrm{C}$ or $13^{\circ} \mathrm{C}-25^{\circ} \mathrm{C}$ for night/day air temperature and $8{ }^{\circ} \mathrm{C}$ or $18^{\circ} \mathrm{C}$ for root temperature. Pollen abortion was observed when air temperature was low $\left(7^{\circ} \mathrm{C}-8^{\circ} \mathrm{C}\right)$ with normal $\left(18^{\circ} \mathrm{C}\right)$ and low $\left(8^{\circ} \mathrm{C}\right)$ root temperature ; "Coldset" was more sensitive than "Montfavet $n^{\circ}$ 63-4". Water uptake was essentially related to air temperature, but was modified by root temperature. The reaction of both genotypes was very similar.

In contrast, the $\mathrm{H}_{2} \mathrm{PO}_{4}^{-}$absorption was related only to root temperature : normal at $18{ }^{\circ} \mathrm{C}$ and halved at $8{ }^{\circ} \mathrm{C}$ for each genotype. $\mathrm{NO}_{3}^{-}$and cation absorption were related to both root and air temperature. There was a difference between both genotypes for $\mathrm{NO}_{3}^{-}$and $\mathrm{K}^{+}$absorption at $8{ }^{\circ} \mathrm{C}$ root temperature.

In summary, no relation appeared between pollen abortion, affected only by low air temperature, and nutrient absorption, that also depended on root temperature.

Additional key words : Lycopersicon esculentum Mill., water supply, mineral nutrition, pollen quality. 


\section{INTRODUCTION}

En cultures froides, sous abris peu ou pas chauffés et en culture précoce de plein champ, la formation des fruits chez la tomate (Lycopersicon esculentum Mill.) est souvent mauvaise. Cette faible nouaison semble due en grande partie à une production insuffisante de pollen fertile (MAISONNEUVE \& PHILOUZE, 1982). La sensibilité variétale pour la production de pollen fertile en serre froide peut être évaluée par un test en conditions contrôlées à $7{ }^{\circ} \mathrm{C}$ (MAISONNEUVE, 1983). Mais dans les conditions du test, où les plantes sont cultivées en pots, la température racinaire est également de $7{ }^{\circ} \mathrm{C}$ alors qu'en serre la température racinaire en zone méditerranéenne est plus élevée. En relation avec l'utilisation de chauffage du sol en serre, il a semblé intéressant d'étudier, en conditions contrôlées, l'effet sur la qualité du pollen de ces températures froides appliquées au niveau racinaire ou au niveau de la partie aérienne. D'autre part, peu d'études ont été faites sur l'action de basses températures appliquées séparément sur les parties aériennes ou racinaires de plantes de tomates. Des températures de sol de $12{ }^{\circ} \mathrm{C}$ associées à des températures d'air assez favorables réduisent la croissance (SHISHIDO \& HORI, 1979) et l'absorption hydrique et minérale (CORNILLON, 1977). Inversement, le chauffage du sol associé à une température nocturne froide de l'air permet de diminuer les conséquences néfastes d'une faible température aérienne sur la croissance et la production (JONES et al., 1978 ; GOSSELIN \& TRUDEL, 1983a). La nutrition hydrique et minérale en conditions froides $\left(7^{\circ} \mathrm{C}\right)$ pour toute la plante ou seulement la partie aérienne n'ayant pas été observée, nous avons entrepris cette étude et nous avons recherché une relation possible entre des déficiences minérales et l'avortement du pollen.

Après avoir fixé les conditions expérimentales, nous présentons les résultats concernant l'influence du rapport entre la température de l'air et celle des racines sur la fertilité du pollen et sur l'absorption hydrique et minérale.

\section{MATÉRIEL ET MÉTHODES}

\section{A. Matériel végétal}

Deux génotypes de tomate à croissance déterminée ont été étudiés : l'hybride F1 «Montfavet $n^{\circ}$ 63-4 » (M) et la lignée «Coldset 》 (C). Des études antérieures ont montré leur différence de sensibilité au froid pour la production de pollen aussi bien en serre non chauffée (MAISONNEUVE \& PHILOUZE, 1982) qu'en conditions contrôlées à $7{ }^{\circ} \mathrm{C}$ (MAISONNEUVE, 1982). De plus, «Montfavet $n^{\circ}$ 63-4 » est très voisin, excepté pour le type de croissance, de l'hybride « Montfavet $n^{\circ}$ 63-5 », dont la nutrition minérale et hydrique a été étudiée à différentes températures racinaires.

\section{B. Méthodes}

\section{Conditions expérimentales}

L'essai a été réalisé sous serre en septembre-octobre dans 4 bacs thermostatés décrits précédemment (RIS-
SER et al., 1978). Les plantes sont élevées en culture hydroponique avec une solution nutritive comprenant par litre : 3,5 meq de $\mathrm{KNO}_{3}, 3,0$ meq de $\mathrm{Ca}\left(\mathrm{NO}_{3}\right)_{2}$, 1 meq de $\mathrm{Mg} \mathrm{SO}_{4}$ et 0,5 meq de $\mathrm{NH}_{4} \mathrm{H}_{2} \mathrm{PO}_{4}$. Cette solution est complétée avec les oligo-éléments essentiels pour la croissance des plantes : $\mathrm{B}, \mathrm{Cl}, \mathrm{Cu}, \mathrm{Fe}$, $\mathrm{Mn}$, Mo et $\mathrm{Zn}$. Le semis est effectué le 31 août 1982 dans un bac de sable quartzeux maintenu à la capacité de rétention en eau et à $18^{\circ} \mathrm{C}$. Le repiquage est réalisé le 16 septembre dans des pots de 1 litre et toutes les plantes sont cultivées à une température de l'air de $13{ }^{\circ} \mathrm{C}-25^{\circ} \mathrm{C}$ nuit/jour et à une température de la solution nutritive de $18{ }^{\circ} \mathrm{C}$. Les traitements thermiques sont effectués dans une $1^{\text {re }}$ expérience sur des plantes de 7 semaines et dans une $2^{\mathrm{e}}$ expérience sur des plantes de 8 semaines, au moment de l'apparition des premières fleurs épanouies sur le premier bouquet. Les températures sont de $7^{\circ} \mathrm{C}-8{ }^{\circ} \mathrm{C}$ (F) nuit/jour ou $13{ }^{\circ} \mathrm{C}-25^{\circ} \mathrm{C}(\mathrm{N})$ nuit/jour pour l'air et de $8{ }^{\circ} \mathrm{C}$ (f) ou $18^{\circ} \mathrm{C}(\mathrm{n})$ pour la solution nutritive. Ces 4 traitements F-f, F-n, N-f, N-n sont effectués pendant 7 j sur 3 plantes par génotype et par expérience; puis les plantes sont replacées en conditions normales $\left(13^{\circ} \mathrm{C}\right.$ $25{ }^{\circ} \mathrm{C}$ pour l'air et $18{ }^{\circ} \mathrm{C}$ pour la solution nutritive). Le refroidissement de l'air est obtenu dans une enceinte de plexiglass ventilée. Les niveaux de températures froides et la durée du traitement ont été choisis d'après des expériences antérieures (MAISONNEUVE, 1982).

\section{Mesures}

\section{a) Qualité du pollen}

La qualité du pollen formé est estimée par le pourcentage de grains d'aspect normal après coloration au carmin acétique chez les fleurs qui s'ouvrent du $11^{\mathrm{e}}$ au $15^{\mathrm{e}} \mathrm{j}$ après la fin du traitement thermique selon la méthode utilisée dans des tests en conditions contrôlées (MAISONNEUVE, 1983). Le pollen est récolté séparément chez 2 fleurs à l'anthèse par plante; 150 grains sont comptés par fleur. Les résultats de chaque fleur sont cumulés pour chaque date de mesure. La moyenne des $5 \mathrm{j}$ de mesure est également calculée.

\section{b) Absorption hydrique et minérale}

La consommation moyenne en eau des plantes de chaque traitement est relevée 2 fois par semaine, avant, pendant et après le traitement en notant les quantités de solution nutritive restantes et celles ajoutées dans les pots de culture. Le renouvellement des solutions nutritives est assuré $3 \mathrm{j}$ avant le traitement, à la mise en place (jour 0 ) et au $4^{\mathrm{e}}, 7^{\mathrm{e}}, 11^{\mathrm{e}}$ et $14^{\mathrm{e}} \mathrm{j}$ pour éviter la prolifération des micro-organismes et pour réduire les variations de concentration en sels.

A chaque renouvellement, des échantillons de solution nutritive sont prélevés et analysés pour déterminer l'absorption globale des anions : $\mathrm{H}_{2} \mathrm{PO}_{4}^{-}$(phosphore), $\mathrm{NO}_{3}^{-}$(nitrate) et des cations : $\mathrm{K}^{+}, \mathrm{Ca}^{++}$, $\mathrm{Mg}^{++}$pour chaque période.

La détermination $\mathrm{du} \mathrm{pH}$ et celle du nitrate sont réalisées avec les électrodes spécifiques correspondantes. Le phosphore est mesuré par colorimétrie au vanado- 
molybdate, le potassium par photométrie de flamme, le calcium et le magnésium par spectrophotométrie d'absorption atomique.

\section{RÉSULTATS}

\section{A. Qualité du pollen}

La figure 1 présente les résultats de la $1^{\text {re }}$ expérience. Le pollen formé dans des conditions de température favorables de l'air $\left(13{ }^{\circ} \mathrm{C}-25^{\circ} \mathrm{C}\right)$ est de très bonne qualité, avec au moins 95 p. 100 de grains normaux, quelle que soit la température de la solution nutritive $\left(18^{\circ} \mathrm{C}\right.$ ou $\left.8^{\circ} \mathrm{C}\right)$. Inversement, quand le pollen est formé à basses températures de l'air $\left(7^{\circ} \mathrm{C}\right.$ $8{ }^{\circ} \mathrm{C}$ ), sa qualité est moyenne ou faible aussi bien pour une température racinaire de $18^{\circ} \mathrm{C}$ que de $8^{\circ} \mathrm{C}$. Des résultats similaires (non présentés) ont été obtenus dans la $2^{\mathrm{e}}$ expérience. Des mesures préliminaires effectuées $7 \mathrm{j}$ après la fin du traitement étaient identiques à celles du $11^{\mathrm{e}} \mathrm{j}$. La moyenne des mesures obtenues pendant $5 \mathrm{j}$ consécutifs montre la plus grande sensibilité de «Coldset » par rapport à « Montfavet $n^{\circ}$ 63-4 » aux 2 températures racinaires (traitements $F-n$ et $F-f$ ).

\section{B. Consommation hydrique et minérale}

Les figures 2 à 6 permettent de suivre au cours du temps l'évolution de la consommation en eau, en phosphore, en nitrate et en potassium pendant le premier essai. Les autres cations $\left(\mathrm{Ca}^{++}, \mathrm{Mg}^{++}\right)$donnent des résultats semblables à ceux du potassium et ne seront donc pas présentés ici. Les résultats sont exprimés en pourcentage du témoin, la variété «Montfavet $\mathrm{n}^{\circ}$ 63-4 » cultivée à des températures normales : $13{ }^{\circ} \mathrm{C}$ $25^{\circ} \mathrm{C}$ nuit/jour pour la partie aérienne et $18^{\circ} \mathrm{C}$ au niveau des racines.

Globalement, nous constatons qu'un choc thermique d'une semaine au niveau des racines et (ou) de la partie aérienne ne provoque pas d'effet néfaste irréversible pour la plante. En effet, une semaine après l'arrêt du traitement, l'absorption de l'eau et des éléments minéraux est redevenue voisine de la normale pour toutes les plantes.

Pendant toute la durée de l'essai, la valeur du $\mathrm{pH}$ de la solution nutritive a varié entre 5 et 7 , ce qui correspond à une plage de valeurs pour laquelle la croissance des plantes demeure normale.

\section{Consommation d'eau}

L'absorption de l'eau dépend essentiellement de la température de l'air pour une même valeur du rayonnement global (fig. 2). Quand la température de l'air est défavorable, la température des racines n'a aucune influence. Par contre, quand elle est favorable, la consommation d'eau est modulée par la température des racines avec un abaissement de la consommation de l'ordre de 30 à 40 p. 100.

Les deux génotypes étudiés réagissent de manière identique aux divers traitements avec de très faibles variations à chaque date de prélèvement. L'abaissement de la température de l'air réduit de 60 à 80 p. 100 l'absorption d'eau.

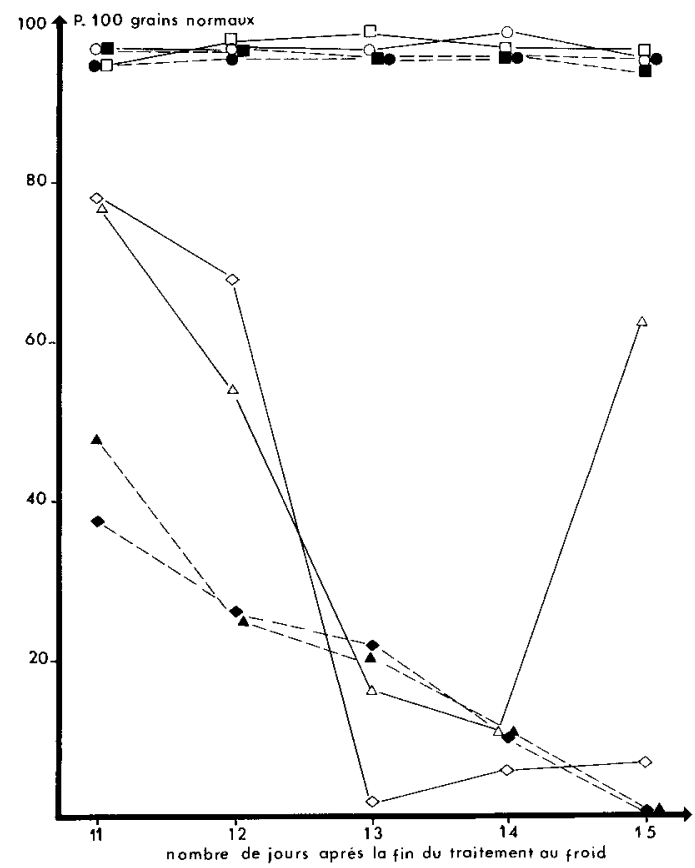

Figure 1

Qualité du pollen ( $p .100$ de grains normaux) chez 2 génotypes après un traitement thermique de 7 jours.

Pollen fertility (\% of normal grains) for 2 genotypes after 7 days of thermal treatment.

\begin{tabular}{|c|c|}
\hline $\begin{array}{l}\text { génotype } \\
\text { | température } \\
\text { rair racine }\end{array}$ & $\begin{array}{l}\text { génotype } \\
\text { température } \\
\text { tair racine }\end{array}$ \\
\hline M F.n $\diamond-$ & C F.n -- \\
\hline M N.n O- & C N.n -- \\
\hline M N.f $\square-$ & C N.f $\square--$ \\
\hline M F.f $\triangle-$ & C F.f $\Delta-$ \\
\hline
\end{tabular}

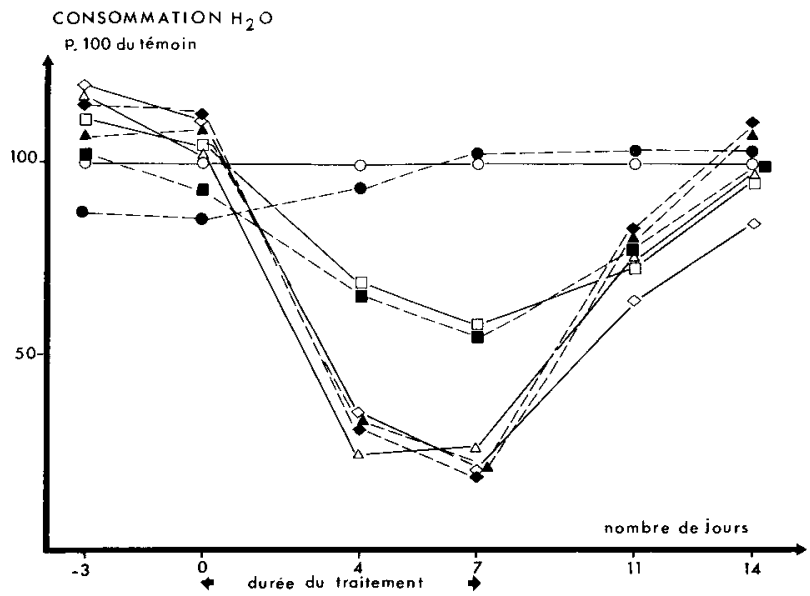

Figure 2

Evolution au cours du temps de la consommation hydrique pour 4 combinaisons de température air-racine chez 2 génotypes $(p .100$ du témoin $M$ 63-4).

Time course of changes in water uptake by 2 genotypes for 4 rootair temperature combinations (\% of M 63-4 as check).

génotype

température

génotype

tair racine

température

M F.n $\diamond-$

MN.nO-

M N.f $\square-$

M F.f $\triangle-$

$$
\text { tair racine }
$$

C F.n

C N.n

C N.f

C F.f 


\section{Absorption du phosphore}

A la concentration de 0,5 meq. $1^{-1}$ de $\mathrm{H}_{2} \mathrm{PO}_{4}^{-}$dans la solution nutritive, l'absorption de cet anion dépend uniquement de la température des racines (fig. 3). La réduction d'absorption paraît d'autant plus grande qu'il existe un déséquilibre important entre la température de l'air et celle des racines.

Il n'apparaît pas d'effet lié au génotype, et quand nous notons la consommation du phosphore en fonction de l'eau consommée, l'allure du phénomène est très voisin pour les 2 génotypes avec les différents traitements.

\section{Absorption du nitrate et des cations}

L'absorption de ces ions dépend de la température des racines et de celle de l'air, le calcium et le magnésium donnant des résultats similaires à ceux du potassium (fig. 4 et 6). Il faut une valeur suffisante de la température au niveau des racines et de la partie aérienne pour observer une nutrition normale. Si l'un des 2 facteurs se situe dans une zone de température défavorable, l'absorption est très affectée.

Le comportement des 2 génotypes, " Montfavet $n^{\circ}$ 63-4» et «Coldset», est relativement uniforme pour tous les traitements en ce qui concerne l'absorption du calcium et du magnésium. Par contre, les 2 génotypes réagissent différemment à la température aérienne de $8{ }^{\circ} \mathrm{C}$ en ce qui concerne la consommation du nitrate (fig. 5) et du potassium par litre d'eau absorbé. Pour « Montfavet $n^{\circ}$ 63-4 », l'absorption du nitrate et du potassium est moins affectée que celle de l'eau. Par contre, pour "Coldset", cette action apparaît plutôt inverse surtout pour le potassium dont la consommation est plus réduite.

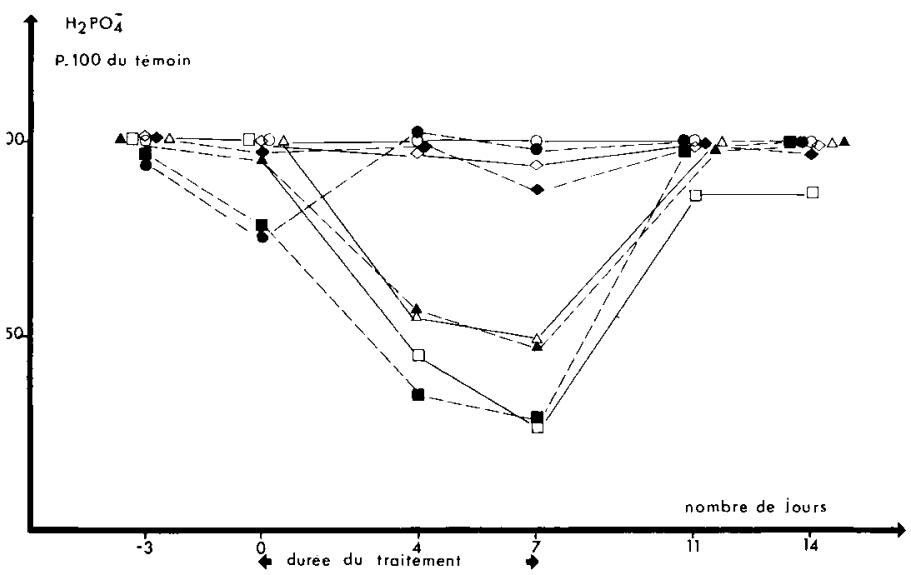

Figure 3

Evolution au cours du temps de l'absorption du phosphate $\left(\mathrm{H}_{2} \mathrm{PO}_{4}\right.$ pour 4 combinaisons de température air-racine chez 2 génotypes (p. 100 du témoin $M$ 63-4).

Time course of changes in phosphorus uptake by 2 genotypes for 4 root-air temperature combinations (\% of M 63-4 as check).

\section{génotype}

température

rair racine

M F.n $\diamond-$

M N.n O-

M N.f $\square-$

M F.f $\triangle$

\section{génotype \\ température \\ rair racine}

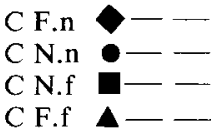

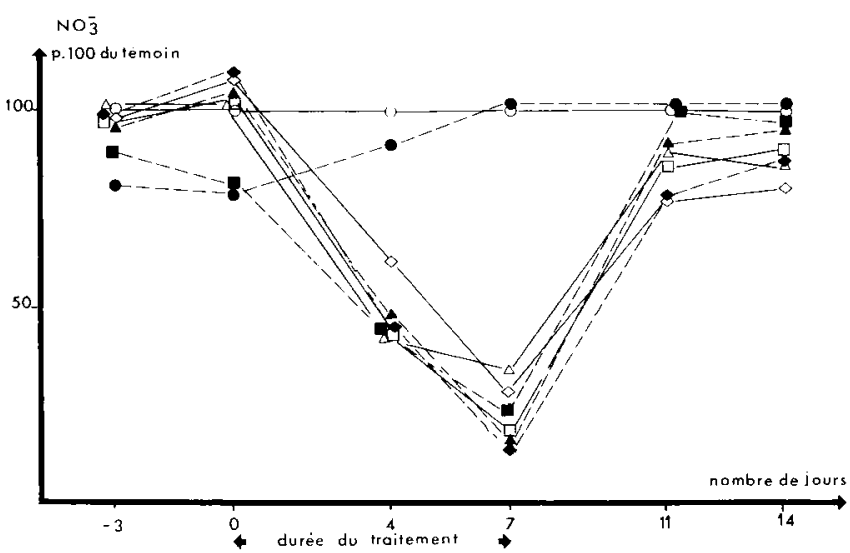

Figure 4

Evolution au cours du temps de l'absorption du nitrate $\left(\mathrm{NO}_{3}^{-}\right)$pour 4 combinaisons de température air-racine chez 2 génotypes (p. 100 du témoin $M$ 63-4).

Time course of changes in nitrate uptake by 2 genotypes for 4 rootair temperature combinations (\% of $M$ 63-4 as check).

génotype

température

génotype

tair racine

température

M F.n $\diamond-$

MN.n O-

M N.f $\square--$

M F.f $\triangle-$

rair racine

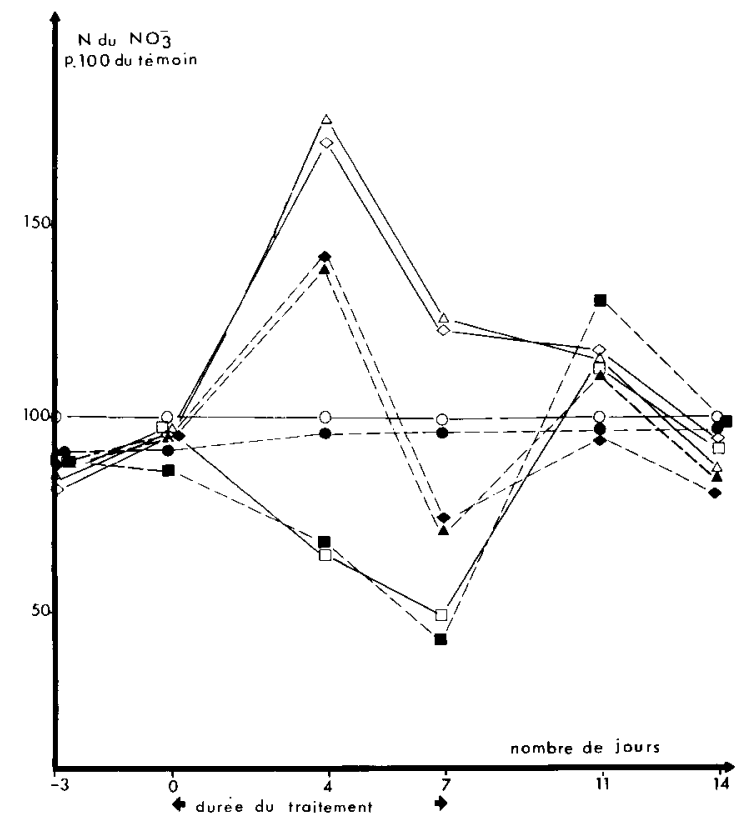

Figure 5

Evolution au cours du temps de l'absorption du nitrate $\left(\mathrm{NO}_{3}^{-}\right)$par litre d'eau consommée par la plante pour 4 combinaisons de température air-racine chez 2 génotypes (p. 100 du témoin M 63-4).

Time course of changes in nitrate uptake per liter of water absorbed by the plant by 2 genotypes for 4 root-air temperature combinations (\% of $M$ 63-4 as check).

\section{génotype}

température

tair racine

M F.n $\diamond-$

$M \mathrm{~N} . n \mathrm{O}-$

$M$ N.f $\square-$

M F.f $\triangle-$ génotype

température

rair racine

$C F n$

C N.n

CNf -

C F.f $\triangle-$ 


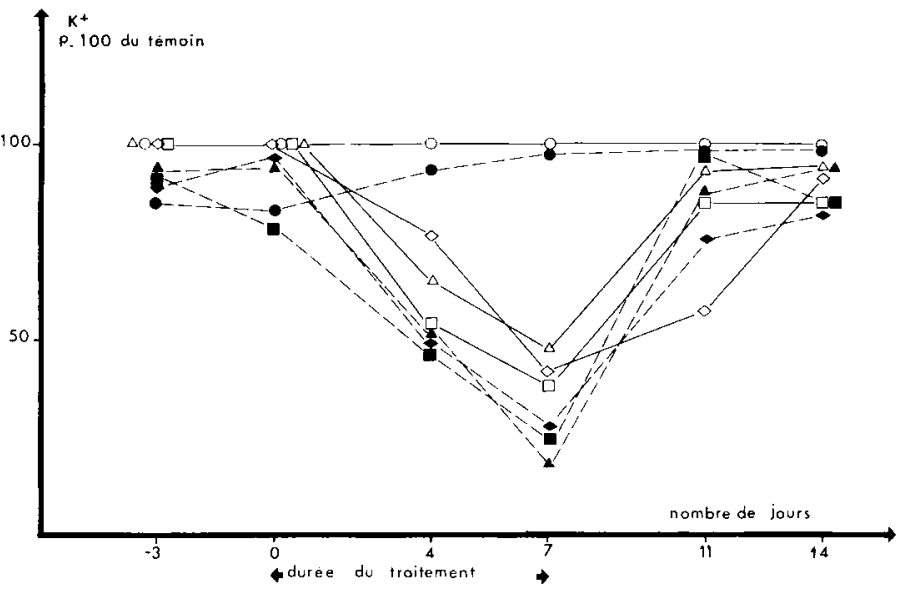

Figure 6

Evolution au cours du temps de l'absorption du potassium $\left(K^{+}\right)$ pour 4 combinaisons de température air-racine chez 2 génotypes (p. 100 du témoin $M$ 63-4).

Time course of changes in potassium uptake by 2 genotypes for 4 root-air temperature combinations (\% of $M$ 63-4 as check) génotype

température

tair racine

M F.n $\bigcirc-$

M N.n O

M N.f $\square-$

M F.f $\triangle-$ génotype

température

tair racine

C F.n

C N.n - -

C N.f - -

C Ff A

\section{DISCUSSION}

\section{A. Qualité du pollen}

Les résultats obtenus en culture froide (F-f) en serre et lumière naturelle concordent avec ceux obtenus en conditions contrôlées (MAISONNEUVE, 1982), le pollen étant sensible aux basses températures 2 semaines avant l'anthèse et "Coldset » étant plus sensible que « Montfavet $n^{\circ}$ 63-4». Les réactions des plantes à chacune des conditions de température aérienne étant identiques pour les 2 niveaux de température racinaire, il semble donc que le froid agit directement sur la partie aérienne pour induire un avortement du pollen. Ainsi, si un chauffage des racines permet une amélioration de la croissance (ABDELHAFEEZ et al., 1971 ; SHISHIDO \& HORI, 1979) ou une limitation du nombre de fleurs (PHATAK et al., 1966) rendu excessif par de basses températures aériennes, il ne peut améliorer la fertilité pollinique et en conséquence la nouaison. Ces résultats sont en accord avec les travaux de CALVERT (1956) selon lesquels un chauffage du sol n'améliore pas la production de tomates cultivées entre $10^{\circ} \mathrm{C}$ et $16^{\circ} \mathrm{C}$ de température nocturne. D'autre part, les résultats obtenus expliquent que le test défini en conditions contrôlées donne une classification variétale semblable à celle d'une serre froide alors que les conditions racinaires sont très différentes.

\section{B. Absorption hydrique et minérale}

Les résultats obtenus au cours de cet essai montrent que l'absorption de l'eau et des éléments minéraux dépend le plus souvent de l'interaction température des racines-température de la partie aérienne. Seule l'absorption du phosphore est liée uniquement à la température des racines.

La demande climatique constitue le moteur essentiel de la consommation en eau mais l'abaissement de la température des racines au-dessous d'un certain seuil réduit fortement l'absorption d'eau. Quand les besoins en eau sont importants, la baisse de la température racinaire doit agir sur l'absorption active à travers le symplasme et sur l'absorption passive à travers l'apoplasme par augmentation de la résistance au transfert de l'eau dans la racine.

KUNG (1981) avait déjà noté l'influence de la température racinaire mais avait observé un effet favorable d'une température nocturne de $10{ }^{\circ} \mathrm{C}$ au niveau de la partie aérienne par rapport à celle de $15^{\circ} \mathrm{C}$. Ainsi, pour les traitements à basse température de l'air, seule la température de $8{ }^{\circ} \mathrm{C}$ pendant la journée jouerait un rôle dans la diminution de l'absorption hydrique.

Le comportement du phosphore apparaît singulier puisque seule la température racinaire agit sur son absorption. Mais le déséquilibre prononcé, entre une température racinaire basse et une température de l'air normale, accentue la mauvaise absorption du phosphore (fig. 3).

L'absorption du nitrate et des cations dépend à la fois de la température de l'air et de celle des racines avec pour le nitrate et le potassium une réaction différente selon le génotype. En ce qui concerne le nitrate, OSMOND \& RAPER (1981) n'avaient pas observé d'interaction entre ces 2 températures pour des plantes de tabac. Mais la gamme de température qu'ils étudiaient était beaucoup plus étroite et elle devait se situer dans une zone où la croissance des plantes est normale. Pour la tomate, une température nocturne de l'air de $10{ }^{\circ} \mathrm{C}$ favorise la nutrition minérale (CORNILLON \& KUNG, 1982). GOSSELIN \& TRUDEL (1983b) ont observé des teneurs élevées en éléments minéraux de la partie aérienne de jeunes plants de tomate quand on maintient des températures relativement élevées au niveau des racines et de l'air pendant la nuit. Mais ce résultat intègre absorption et migration des éléments minéraux.

Pour des plantes dont la croissance s'est effectuée dans des conditions normales de nutrition jusqu'à la floraison du premier bouquet, une semaine de traitement à très basse température s'avère insuffisante pour provoquer l'apparition de symptômes de carence.

\section{Relation entre l'absorption racinaire et la qualité du pollen}

Dans ces conditions expérimentales, il ne semble pas y avoir de relation entre l'absorption de certains ions minéraux $\left(\mathrm{H}_{2} \mathrm{PO}_{4}^{-}, \mathrm{NO}_{3}^{-}, \mathrm{K}^{+}, \mathrm{Ca}^{++}\right.$et $\left.\mathrm{Mg}^{++}\right)$et la microsporogenèse. En effet, en conditions de faible absorption du phosphore, du nitrate et des cations, la qualité du pollen formé peut être bonne (traitement $\mathrm{N}-\mathrm{f}$ ). La réaction des plantes aux différents traitements est même parfaitement opposée en ce qui concerne l'absorption du phosphore et la fertilité pollinique. Ainsi, si des déficiences en phosphore, calcium et magnésium diminuent la taille des grains de pollen (BELL, 1959), elles ne provoquent pas de blocage de la 
microsporogenèse. Il serait intéressant d'analyser le devenir du bore puisque cet élément semble exercer une action sur la germination du pollen d'après des études de germination in vitro. La comparaison des 2 génotypes conduit aux mêmes conclusions puisque " Coldset » est plus sensible au froid que « Montfavet $n^{\circ}$ 63-4 » en ce qui concerne la microsporogenèse, mais nous n'observons pas de différences variétales pour ce qui est de l'absorption racinaire du phosphore, du calcium et du magnésium.

\section{CONCLUSION}

L'action de la température des racines et celle de la partie aérienne varient selon le mécanisme étudié : qualité du pollen ou absorption hydrique et minérale de la plante.

Quand on soumet des plantes à un traitement thermique d'une semaine au moment de l'apparition des premières fleurs épanouies, la qualité du pollen est seulement sensible aux basses températures de l'air, tandis que l'absorption du phosphore est sous la dépendance unique de la température racinaire. L'absorption de l'eau, du nitrate et des cations dépend à la fois de ces 2 facteurs.

Ainsi le chauffage du sol améliore l'absorption du phosphore, mais il ne peut pas empêcher la mauvaise nouaison des tomates due à l'avortement du pollen ce qui ne permet pas de substituer le chauffage des serres par le sol à celui de l'air. Cette différence de comportement fournit une explication à la meilleure production obtenue quand on utilise le paillage radiant par rapport à celle notée avec le chauffage du sol par tuyaux enterrés (WACQUANT et al., 1979).

Il apparaît qu'une température suffisante doit être maintenue au niveau des racines et de la partie aérienne pour assurer une production normale grâce à une bonne fertilité pollinique et à une nutrition convenable.

Reçu le 2 février 1984. Accepté le 17 juillet 1984.

\section{RÉFÉRENCES BIBLIOGRAPHIQUES}

Abdelhafeez A. T., Harssema H., Veri G., Verkerk K., 1971. Effects of soil and air temperature on growth, development and water use of tomatoes. Neth. J. agric. Sci., 19, 67-75.

Bell C. R., 1959. Mineral nutrition and flower to flower pollen size variation. Am. J. Bot., 46 (9), 621-624.

Calvert A., 1956. The influence of soil and air temperatures on cropping of glasshouse tomatoes. J. Hortic. Sci., 31, 69-75.

Cornillon P., 1977. Effet de la température des racines sur l'absorption des éléments minéraux par la tomate. Ann. Agron., 28 (4), 409 423.

Cornillon P., Kung N., 1982. Influence de la température des racines et de la température ambiante nocturne sur l'absorption des éléments minéraux par la plante. Acta Hortic., 126, 31-35.

Gosselin A., Trudel M. J., 1983a. Interactions between air and root temperatures on greenhouse tomato : I Growth, development, and yield. J. Am. Soc. Hortic. Sci., 108 (6), 901-905.

Gosselin A., Trudel M. J., 1983b. Interaction between air and root temperatures on greenhouse tomato : II Mineral composition of plants. J. Am. Soc. Hortic. Sci., 108 (6), 905-909.

Jones D. A. G., Sandwell I., Talent C. J. W., 1978. The effect of soil temperature when associated with low air temperatures on the cropping of early tomatoes. Acta Hortic., 76, 167-171.

Kung N., 1981. Effets conjugués de la température nocturne de l'air et de la température au niveau des racines sur le comportement de la tomate. D.A.A., ENSA Toulouse, 41 p.

Maisonneuve B., 1982. Effet d'un traitement à basses températures, en conditions contrôlées, sur la qualité du pollen de tomates ( $L y c o-$ persicon esculentum Mill.). Agronomie, 2 (8), 755-764.

Maisonneuve B., 1983. Qualité du pollen formé au froid chez la tomate (Lycopersicon esculentum Mill.). I Amélioration d'un test en conditions contrôlées. Agronomie, 3 (9), 873-878.

Maisonneuve B., Philouze J., 1982. Action des basses températures nocturnes sur une collection variétale de tomate (Lycopersicon esculentum Mill.). II Etude de la quantité et de la qualité du pollen. Agronomie, 2 (5), 453-458.

Osmond D. L., Raper J. C. D., 1981. Growth and nitrogen accumulation in tobacco plants as affected by nitrate concentration, root temperature and aerial temperature. Agron. J., 73 (3), 491-496.

Phatak S. C., Wittwer S. H., Teubner F. G., 1966. Top and root temperature effects on tomato flowering. Proc. Am. Soc. Hortic. Sci., 527-531.

Risser G., Cornillon P., Rode J.-C., Auge M., 1978. Effet de la température des racines sur la croissance de jeunes plants de diverses variétés de melon (Cucumis melo L.). Ann. Agron., 29 (5), 453473.

Shishido Y., Hori Y., 1979. Studies on translocation and distribution of photosynthetic assimilates in tomato plants. III Distribution pattern as affected by air and root temperatures in the night. Tohoku J. Agric. Res., 30 (3), 87-94.

Wacquant C., Musard M., Rassent E., Fabry J., Odet S., 1979. Etude des effets de deux systèmes de chauffage utilisant les rejets thermiques de centrales sur la production de printemps de tomate sous serre plastique. C.T.I.F.L., 486-71, $33 \mathrm{p}$. 